\title{
Local Loss Coefficient of Laminar Flow through Sudden Enlargement in Circular Pipes
}

\author{
Y. B. Wu ${ }^{1 *}$, B. Zhang ${ }^{1 *}$, J. W. Xiao ${ }^{2}$ \\ ${ }^{1}$ School of Municipal and Environmental Engineering, Harbin Institute of Technology, Harbin 150090, \\ China; \\ 2 Shenzhen Zhonghai Construction Co. Ltd, Shenzhen, China \\ Email: ybwu@hit.edu.cn, zhangbei15@isina.com
}

\begin{abstract}
Generally local loss coefficient is found to be just the function of enlargement ratio at sudden enlargement in circular pipes in turbulent flow. However, in many other instances, the Reynolds number is low, and the flow is laminar. Then local loss coefficient varies significantly not only with enlargement ratio but also with Reynolds number. Therefore, the existing results lack accuracy in the laminar regime.

In literatures, some study is presented about local loss coefficients of laminar flow through sudden enlargement in circular pipes, but they are limited to the only case of a uniform inlet velocity profile. Oliveira [1] reported the pressure loss coefficients taking account of the full developed conditions but all results were limited to the enlargement ratio $E=2.6$. In this paper, numerical simulation of the laminar flow has been carried out at enlargement ratios $E=1.5,2,3,4$ and 5 and Reynolds numbers below 225 .

The laminar flow in axisymmetric sudden cnlargement is studied by solving the Navier-Stokes cquation using the finite volume method. The SIMPLER algorithm based on staggered grid is implemented with Fortran 90. The results of the numerical analysis are quantified in terms of the reattachment length and compared with available cxperimental data in literatures. Good agreement is found. So the comparisons deem sufficiently validate all aspects of the calculation procedure and convince us its capacity to analyze the local loss based on the simulation results. Local loss coefficicnts at enlargement ratio of $1.5,2,3,4$ and 5 are calculated respectively at Reynolds numbers below 225 . From the trend of the coefficients varying with the Reynolds number, we can find that at low Reynolds numbers $(R e<10)$ the local loss coefficient is inversely proportional to Reynolds number and nearly not affected by enlargement ratio since the flow is dominated by viscous forces. The results are compared with experimental data in literature [2] and good agreement is found. At moderate Reynolds numbers the local loss coefficient is a function of both Reynolds number and enlargement ratio. With the Reynolds number increasing gradually the local loss coefficient tends to be constant and is just a function of the enlargement ratio as in turbulence.
\end{abstract}

\section{REFERENCES}

1. Oliveira P. J. Pressure drop coefficient of laminar Newtonian flow in axisymmetric sudden expansions. International Journal of Heat and Fluid Flow, 1997;18:518-529

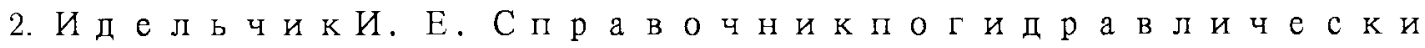
м. M, М а ши н о о с т р о е н и е, 1975 